\title{
Transition Radiation Detector in the D $\emptyset$ Colliding Beam Experiment at Fermilab
}

\author{
Henryk Piekarz \\ For the D $\varnothing$ Collaboration \\ Florida State University \\ Tallahasse, Florida 32306 \\ Fermi National Accelerator Laboratory \\ P.O. Box 500, Batavia, Illinois 60510
}

April 1995

Presented at the Vienna Wire Chamber Conference 95, Vienna, Austria, February 13-17, 1995 


\section{Disclaimer}

This report was prepared as an account of work sponsored by an agency of the United States Government. Neither the United States Government nor any agency thereof, nor any of their employees, makes any warranty, express or implied, or assumes any legal liability or responsibility for the accuracy, completeness, or usefulness of any information, apparatus, product, or process disclosed, or represents that its use would not infringe privately owned rights. Reference herein to any specific commercial product, process, or service by trade name, trademark, manufacturer, or otherwise, does not necessarily constitute or imply its endorsement, recommendation, or favoring by the United States Government or any agency thereof. The views and opinions of authors expressed herein do not necessarily state or reflect those of the United States Government or any agency thereof. 


\title{
TRANSITION RADIATION DETECTOR IN THE DO COLLIDING BEAM EXPERIMENT AT FERMILAB
}

\author{
HENRYK PIEKARZ* \\ Florida State University, Tallahasse, \\ Florida, 32306, U.S.A.
}

A presentation at Vienna Wire Chamber Conference '95

Vienna, February 13-17, 1995

"Representing the DØCollaboration at Fermi National Accelerator Laboratory, Batavia Ill 60510. 


\title{
TRANSITION RADIATION DETECTOR IN THE DO COLLIDING BEAM EXPERIMENT AT FERMILAB
}

\author{
HENRYK PIEKARZ* \\ Florida State University, Tallahasse, \\ Florida, 32306, U.S.A.
}

\begin{abstract}
The construction, operation and response of the Transition Radiation Detector (TRD) at D0 colliding beam experiment at Fermilab are presented. The use of the TRD signal to enhance electron identification and hadronic rejection in the multiparticle background characteristic for the antiproton-proton interactions at the center-of-mass energy of $1.8 \mathrm{TeV}$ is also described and results are discussed.
\end{abstract}

Transition radiation (TR) $x$-rays ${ }^{[1]}$ are produced when a relativistic particle traverses a boundary of two media with differing dielectric constants. The TR photons are detected in a gas of high $\mathrm{Z}$ (such as Xenon) in order to improve the energy ratio of the TR $\mathrm{x}$-rays with respect to the ionization processes. Only highly relativistic particles can produce TR signal which strength is comparable to that of the ionization alone. In practical applications the TR detectors are mostly used to identify electrons with $\gamma$ $\geq 210^{3}$ ( $\left.\mathrm{E} \geq 1 \mathrm{GeV}\right)$.

The D0 TRD detector and its location with respect to the central tracking detectors and calorimeter is shown in Fig.1. The figure represents 1/4 of the D0 detector cross-section. The TRD detector is located between the vertex and the central tracking chambers. The calorimeter is outside the central tracking detectors. The electrons and hadrons which originate in the beam interaction region traverse the tracking detectors and deposit energy in the calorimeter. Extensive studies and prototype tests were done to optimize the TRD detector performance at D0 colliding beam experiment and to simplify the construction techniques of the device. The tests results and the details of the construction were described in previous papers ${ }^{[2,3,4,5]}$.

The TRD detector at D0 consists of three chambers. A cross-section of the TRD chamber is shown in Fig.2. Its total radial width is $11 \mathrm{~cm}$. The structure of the TRD chamber is supported by two $1 \mathrm{~cm}$ thick plastic honeycomb cylinders with fiber glass skins. In order to optimize the production and the detection of TR photons, the TRD chamber consists of two principal compartments: (1) a radiator, where the TR photons

* Representing the DØCollaboration at Fermi National Accelerator Laboratory, Batavia Ill 60510. 
are produced and (2) a detector, where the TR photons are detected. The radiator is 6.5 $\mathrm{cm}$ wide in the radial direction and it consists of 400 foils of $18 \mu \mathrm{m}$ thick polypropylene, housed in the nitrogen filled enclosure. The mean gap between the foils is about 150 $\mu m$. The detector is a two-stage time-expansion radial drift/PWC chamber filled with Xenon-Methane gas mixture (91\%-9\%). TR photons produced in radiator convert to electrons in the first zone of the detector and the resulting charge drifts radially outward to the anode wires where the avalanche occures. The drift zone is $15 \mathrm{~mm}$ wide and the amplification zone is $8 \mathrm{~mm}$ wide. The two zones are seperated with cathode wire grid of Au plated Tungsten wire $70 \mu m$ thick. The anode wires in the amplification zone are Au plated Tungsten wires $30 \mu \mathrm{m}$ thick. The potential wires are $\mathrm{CuBe}, 100 \mu \mathrm{m}$ thick. There are 256 anode wires in chamber 1 and 2, and 512 anode wires in chamber 3 . In addition, the rear cathode of the amplification zone is made of helical $\mathrm{Cu}$ strips (256 for chamber 1 and 2, and 512 for chamber 3) deposited on the Kapton foil; the pitch angle varies between $24^{\circ}$ and $47^{\circ}$. The detector and radiator compartments are separated by a $0.2 \mathrm{~cm}$ wide gap compartment filled with carbon dioxide. The primary role of the gap is to diminish the permeation of nitrogen from the radiator into the detector compartment. The aluminized gap window facing the detector is on the most negative potential thus forcing in this way the ionization electrons to drift into the amplification zone of the detector. The thickness of the full TRD detector at $\Theta=90^{\circ}$ is $8.1 \%$ radiation length and $3.6 \%$ of the interaction length thus causing only small scattering or interaction of particles traversing it.

The charge collected from both, the anodes and the cathodes is read out. The signal from the cathodes is about three times smaller than that of the anodes as there is no amplification at the cathode. The electronics readout chain is shown in Fig.3. The charge sensitive amplifier is connected to the anode wire or the cathode strip of the TRD detector. The signal is then sent to the shaper module where after amplification it passes through the cable compensation and frequency response shaping. These networks provide gain in the frequency range where the cables attenuate the signal as well as they shape the signal into a more Gaussian form. The anti-aliasing filter in the Flashing Analog to Digital Converter (FADC) module is a low-pass filter with a corner frequency less than the Nyquist frequency thus helping to reduce distortions in the digitization process. There are total of 2048 readout channels in the D0 TRD detector.

The photon conversion depth in the TRD detector is energy dependent but as most of TR photons are expected to convert in first few millimiters of the detector compartment, well isolated energy clusters should be observed due to passing electrons. This is in contrast to the ionization electrons which are produced along the entire particle path. The characteristic FADC output for the electron candidate track is shown in Fig.4. The vertical scale represents the cluster energy while the horizontal one the cluster arrival time. One can see that in all three TRD chambers well isolated, energetic clusters were detected thus indicating that indeed this track is a candidate for an electron.

The TRD detector response depends on the pressure, temperature and purity of the xenon gas mixture. The gas pressure in the TRD chamber has the strongest impact 
on the response. As the exterior walls as well as those separating the detector, gap and radiator compartments are very thin to minimize the scattering of particles and TRD $x$-rays absorption, the gas in the TRD chambers must be regulated with respect to the atmospheric pressure. In addition, the high cost of the xenon gas requires that the TRD gas mixture must be recirculated. In order to satisfy the above conditions a rather sophisticated, automatically controlled gas system was designed for the D0 TRD detector. The xenon gas pressure in the chambers is regulated within about 0.04 Torr relative to the atmospheric pressure. The response time of the system to the atmospheric pressure change is $100 \mathrm{msec}$. The air and carbon dioxide can contaminate the TRD chambers due to leaks and gas permeation through the dividing walls. The oxygen and carbon dioxide which may most strongly affect the TRD detector response are continuously trapped in the designated filters. The oxygen contamination level is typically below $2 p p m$.

The purity of the TRD gas mixture is continuously tested using a designated Canary test chamber located at the gas exit from the chambers. The changes in TRD detector response, however, are monitored using the detection of the radiation emitted from the uranium plates in the calorimeter. This radiation produces a fluorescent line in the Xenon at about $30 \mathrm{keV}$. The energy of this line is rather at the end of the energy range of the clusters produced by high energy hadrons or electrons traversing TRD chamber and it is about twice the energy deposited by a minimum ionizing particle. A characteristic "uranium noise" spectrum observed in a TRD chamber is shown in Fig.5. In Fig.6 we present a change of the response for all three TRD chambers during recent 200 days of the collider Run $1 \mathrm{~b}$. This response was corrected for pressure and temperature changes of the TRD gas mixture. The integrated luminosity in this period of time was about $60 \mathrm{pb}^{-1}$. We observe the loss of the response of about $1 \% / 4 p b^{-1}$ or about $7 \% / 100$ days. The typical anode currents at luminosity of $10^{31}$ are in the range of $(50-60) \mu \mathrm{A}$. For 200 days of the colliding beam experiment the integrated luminosity was of the order of $210^{38}$, and thus the total accumulated charge was about $310^{-2}$ Coulomb $/ \mathrm{cm} /$ wire. This accumulated charge is not very high, so it does not entirely explains the observed loss of the signal. A possibility that some undetected TRD gas contamination caused the loss of the signal is also ruled out as several total xenon mixture purges produced only very minor improvement in the TRD response. The increase of the instantaneous luminosity from $510^{30}$ range to about $10^{31}$ caused the $\mathrm{HV}$ anode currents to increase to the values exceeding currents at lower luminosity by a factor of 3 to 5 . The increased moisture level in the TRD gas mixture from 1500 $p p m$ to about $6500 \mathrm{ppm}$ reduced however these anomalous anode currents to the level expected for the luminosity of $10^{31}$.

In order to test the ability of TRD to identify electrons we use a sample of $W \rightarrow e \nu$ decay candidate events and compare with the minimum bias events which are strongly dominated by hadrons. The electron energies in $W \rightarrow e \nu$ candidate events are selected using a minimum transverse energy of the calorimeter cluster of $25 \mathrm{GeV}$. In the D0 detector the calorimeter provides primary information on the hadronic or electromagnetic origin of the observed shower. The track information from the central 
tracking detectors allows one to select a "road" in which the TRD signal should be examined. Using this method the TRD observed energy spectra for both, $W \rightarrow e \nu$ and minimum bias events are shown in Fig.7. One can see that while the energy deposits for the minimum bias events peak strongly at low energies there exists a long tail of minimum bias events with energies overlapping those due to electrons from the $W \rightarrow e \nu$ decays. By requesting, however, a TRD signal from 3 detector layers simultaneously the probability of identifying electron and rejecting a hadron can be greatly improved. Using information from all three TRD detector layers the probability of being electron (or hadron) can be calculated for each electron candidate event. A Neyman-Pearson test has been developed using the distributions of energy deposited in the 3 chambers for the electrons and for the minimum bias tracks (hadrons). This test corresponds to computation of the ratio $R$ of the Likelihood values for the electron and the hadron hypothesis. The distributions of $\log (R)$ for the electron candidates from the $W \rightarrow e \nu$ data sample and hadrons from the minimum bias events are shown in Fig.8. The most probable Likelihood values for electrons and hadrons are very well separated but there exists significant overlap which indicates that higher identification probability must be accompanied by loss of efficiency. The electron detection efficiency versus the rejection of the minimum bias tracks from the hadronic background is shown in Fig. 9. For a 5 -fold hadronic rejection power the electron detection efficiency is about $90 \%$.

The Likelihood distribution for electron candidates in the top quark candidate event sample observed in the D0 detector is shown in Fig.10. The actual events used in the top quark analysis are shown in black. One can see that the TRD Detector at D0 provided very useful information for final identification of the top quark candidate events.

\section{References}

1. V.L.Ginzburg and I.M.Franck, Zh.EXp.Th Phys., 16 (1946) 15.

2. J.F.Detoeuf et al., Nucl. Instr. and Meth., A265 (1988) 157.

3. J.F.Detoeuf et al., Nucl. Instr. and Meth., A279 (1989) 310.

4. Y.Ducros et al., Nucl. Instr. and Meth., A277 (1989) 401.

5. S.Abachi et.al., Nucl.Instr. and Meth., A338 (1994) 185. 


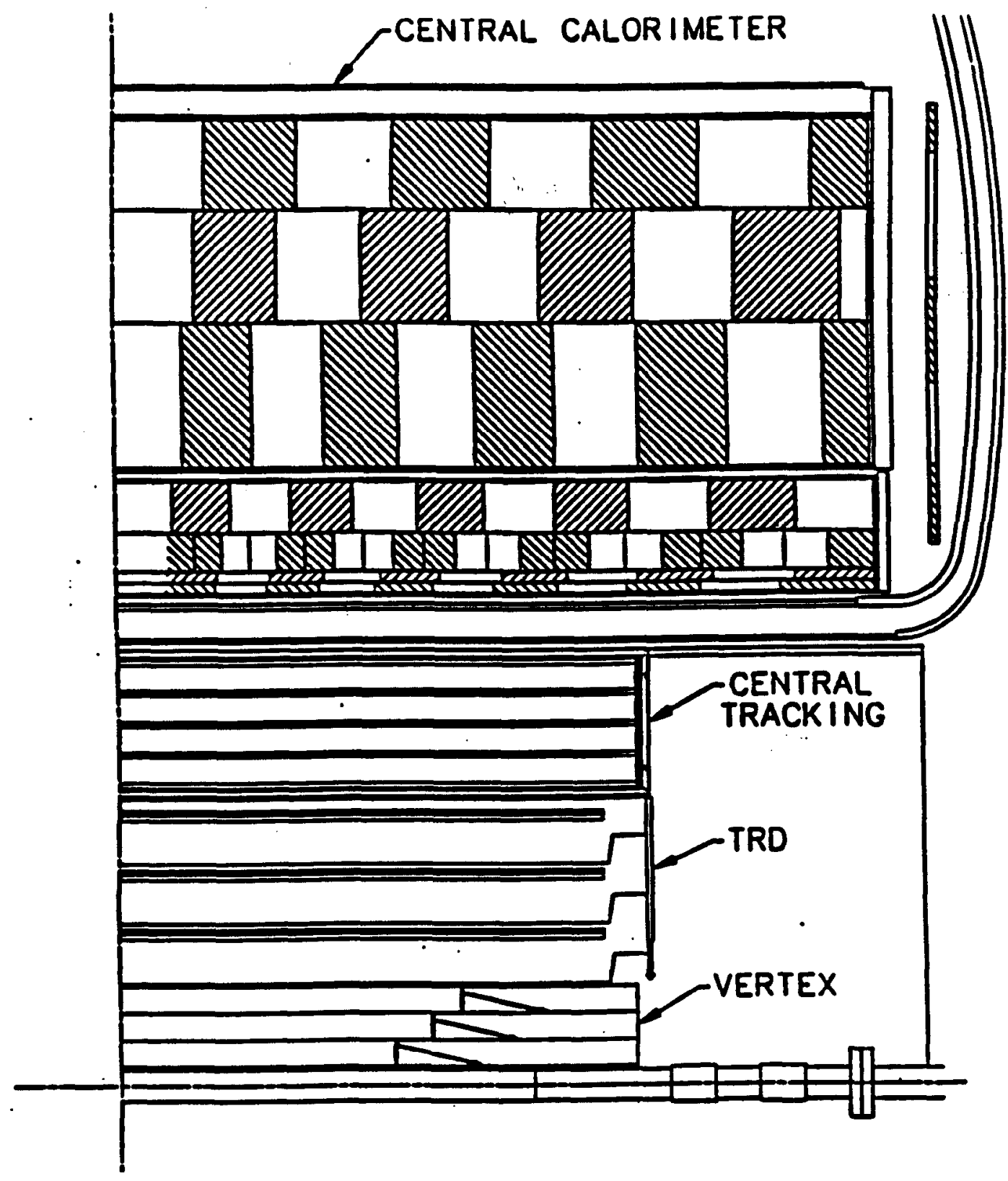

Fig. 1 The location of the TRD detector with respect to central tracking chambers and calorimeter in the D0 colliding beam experiment. 


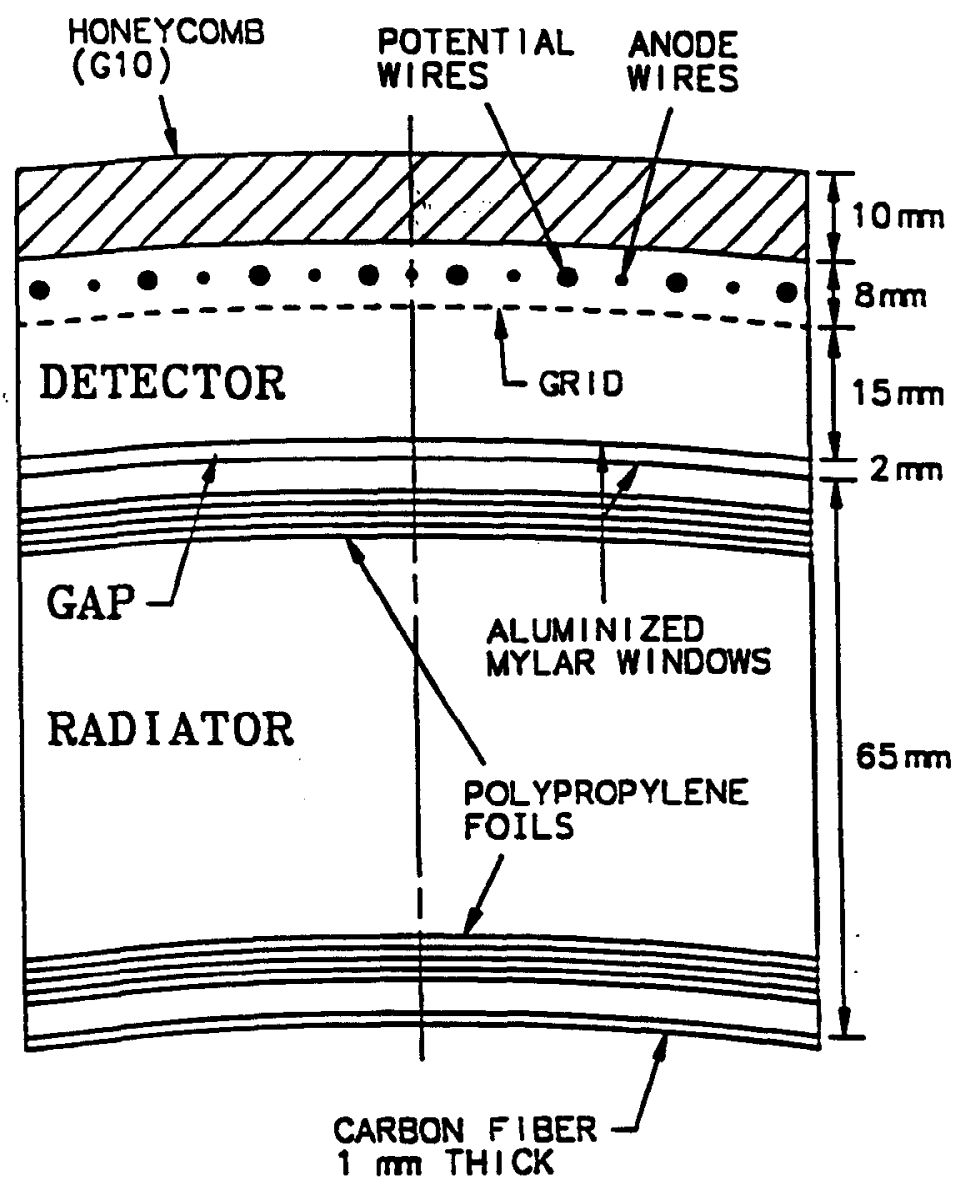

Fig.2 The cross-section of a typical cell in the D0 TRD detector. 


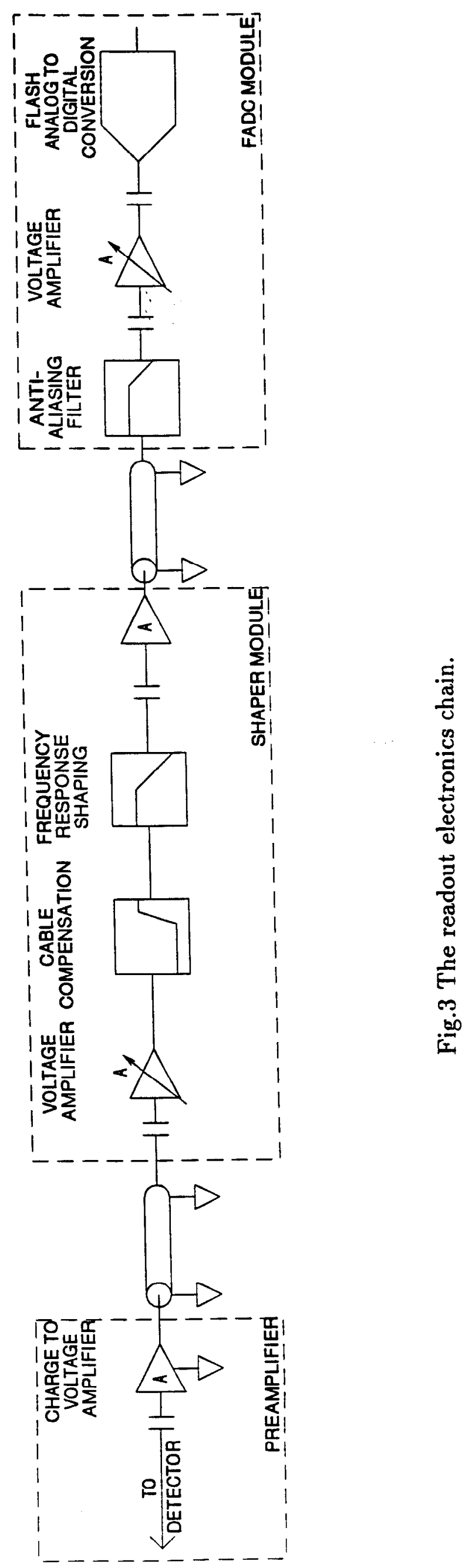



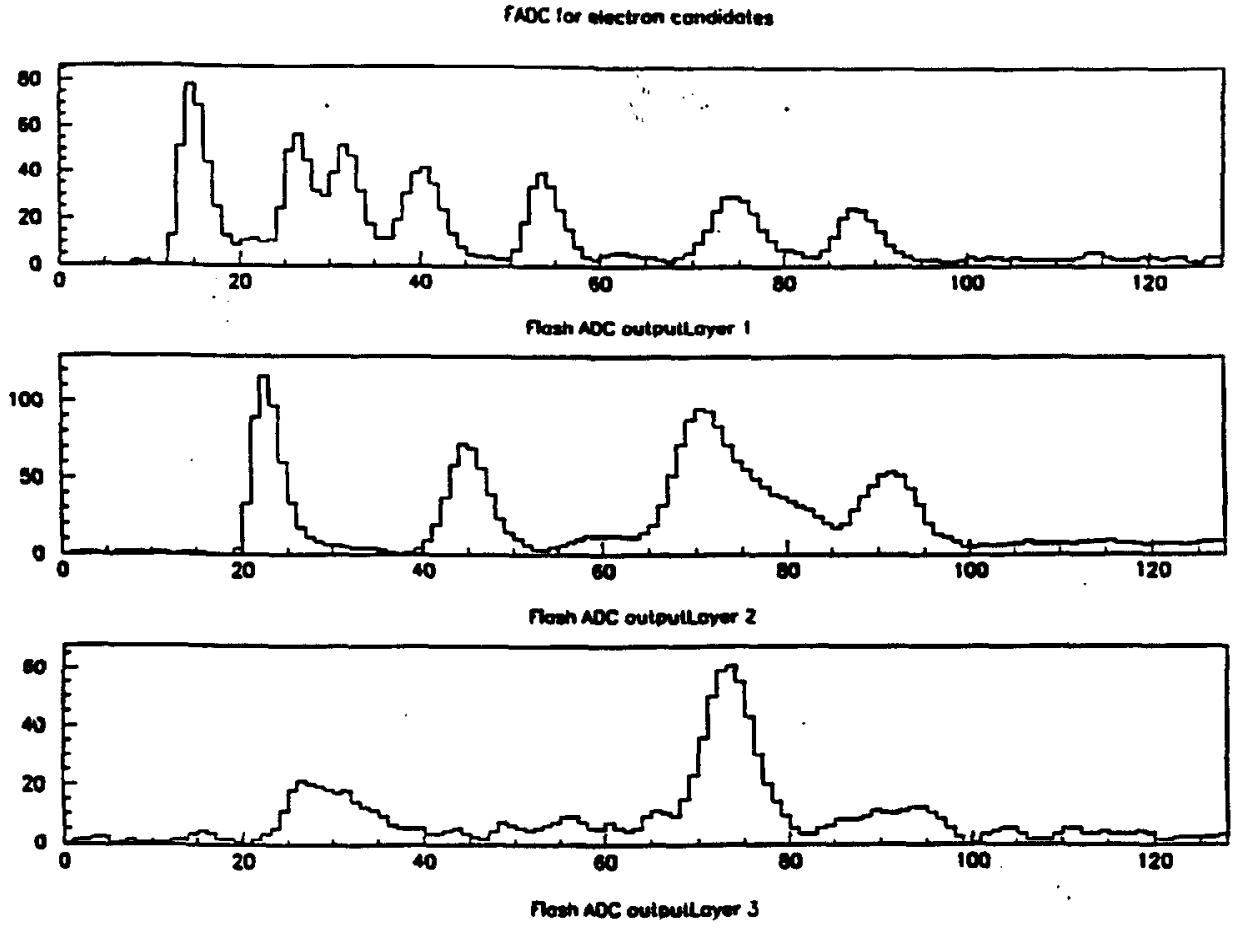

Fig.4 The typical signal and noise in the TRD FADC. 

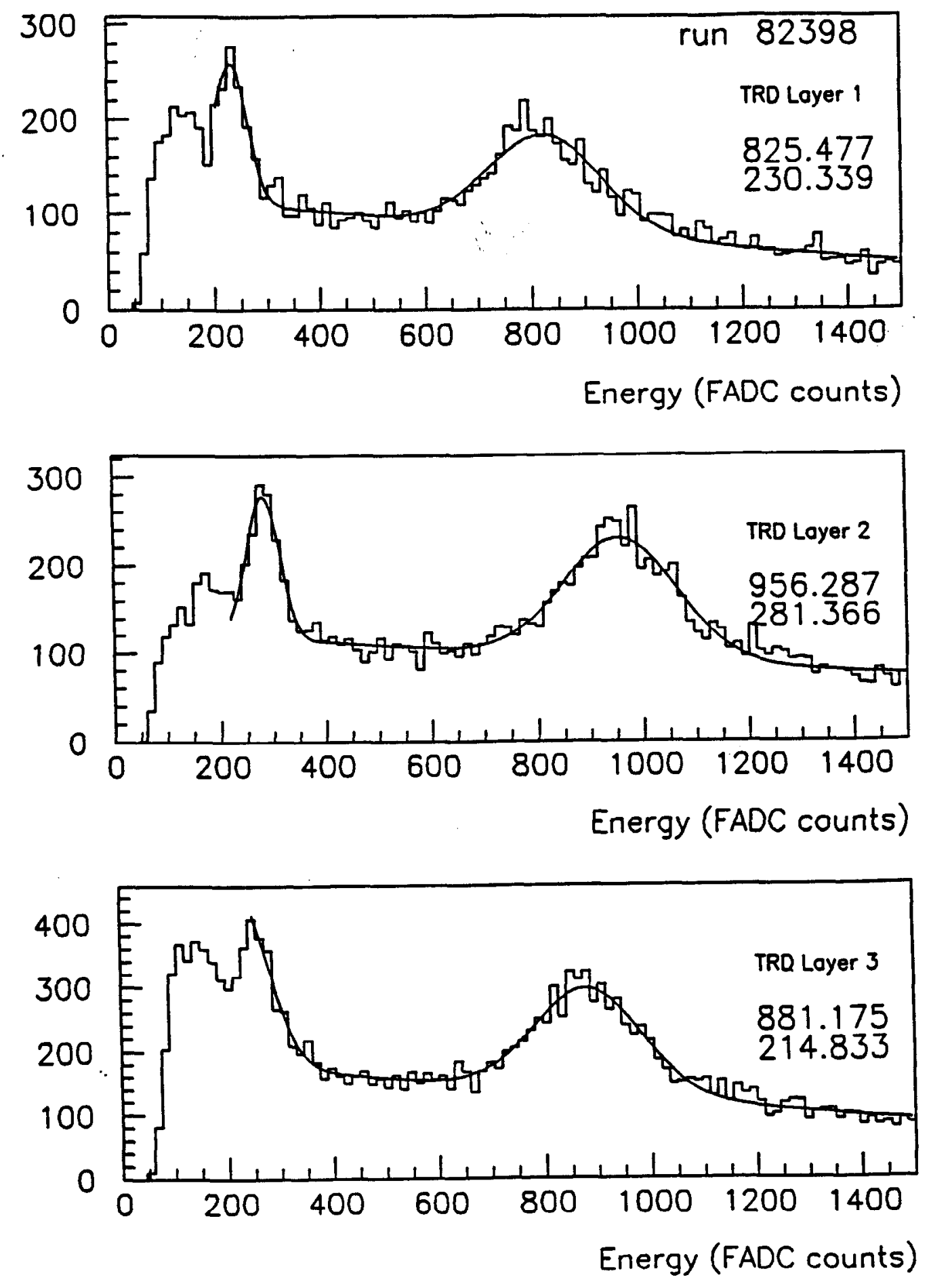

Fig.5 The TRD energy spectrum due to the D0 calorimeter uranium radiation. 

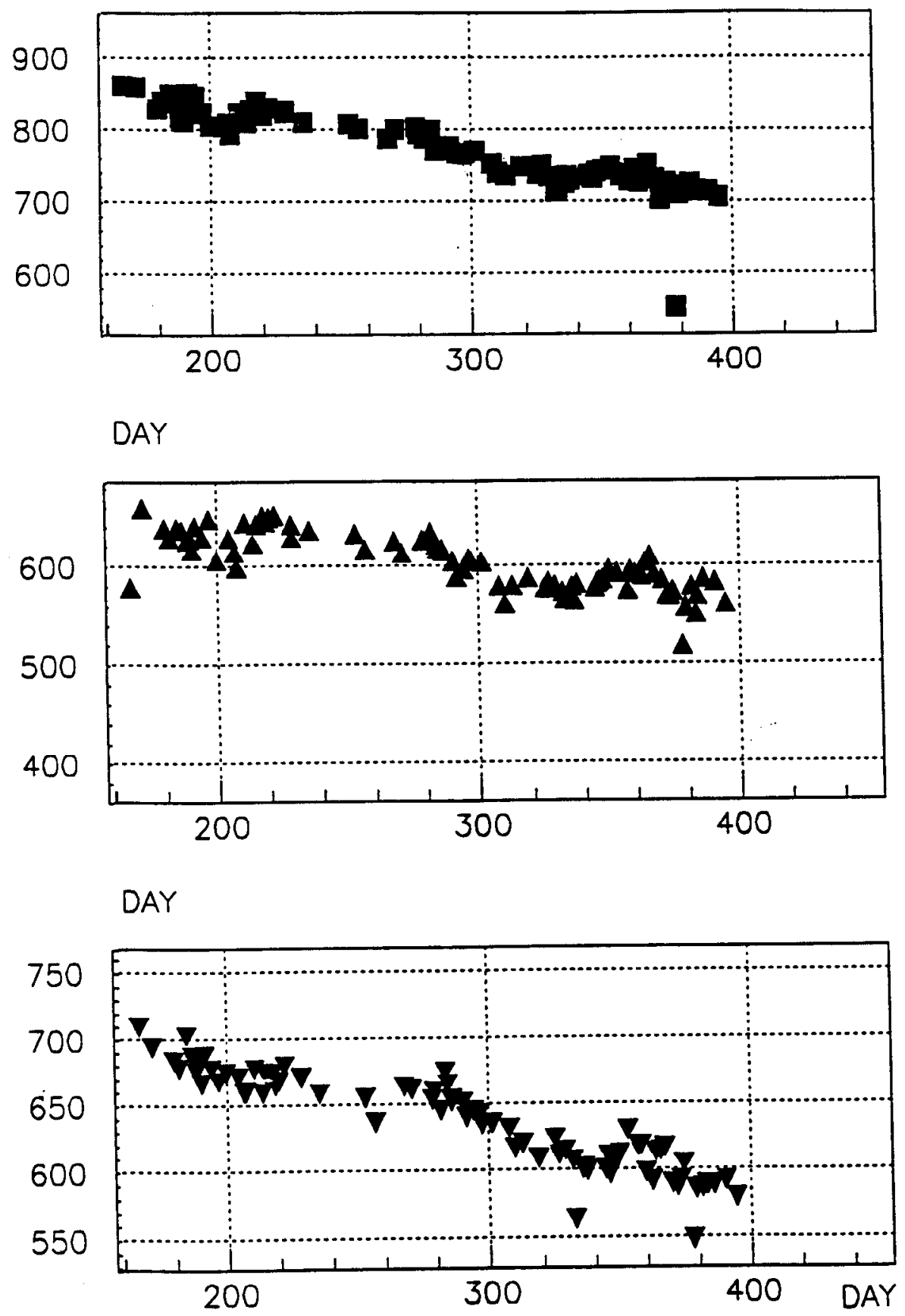

Fig.6 The TRD detector response to uranium radiation as a function of time during the collider Run $1 \mathrm{~b}$. 

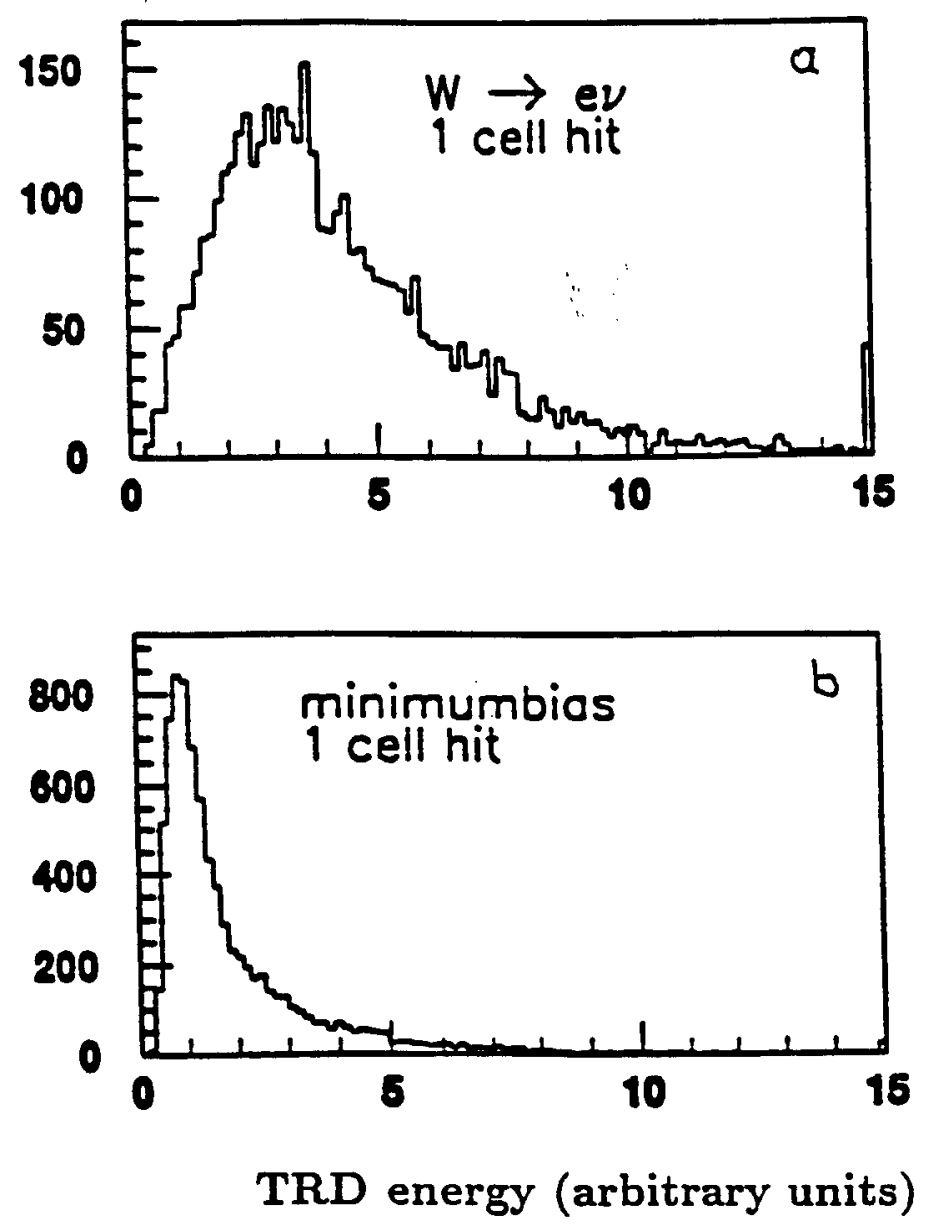

Fig.7 The TRD energy spectra for: (a) electrons from the $W \rightarrow e \nu$ decays and (b) hadrons from the minimum bias events. 


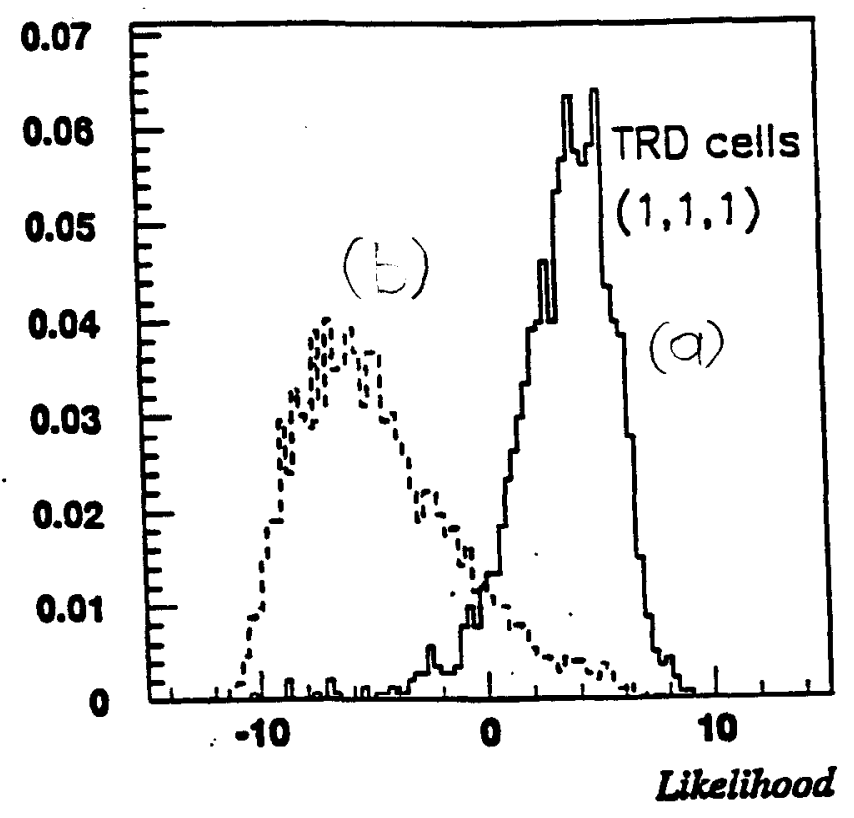

Fig. 8 The distribution of the Likelihood function for: (a) electrons from the $W \rightarrow e \nu$ decays and (b) hadrons from the minimum bias events. These Likelihood distributions were obtained using the combined TRD and central tracking chambers information. 


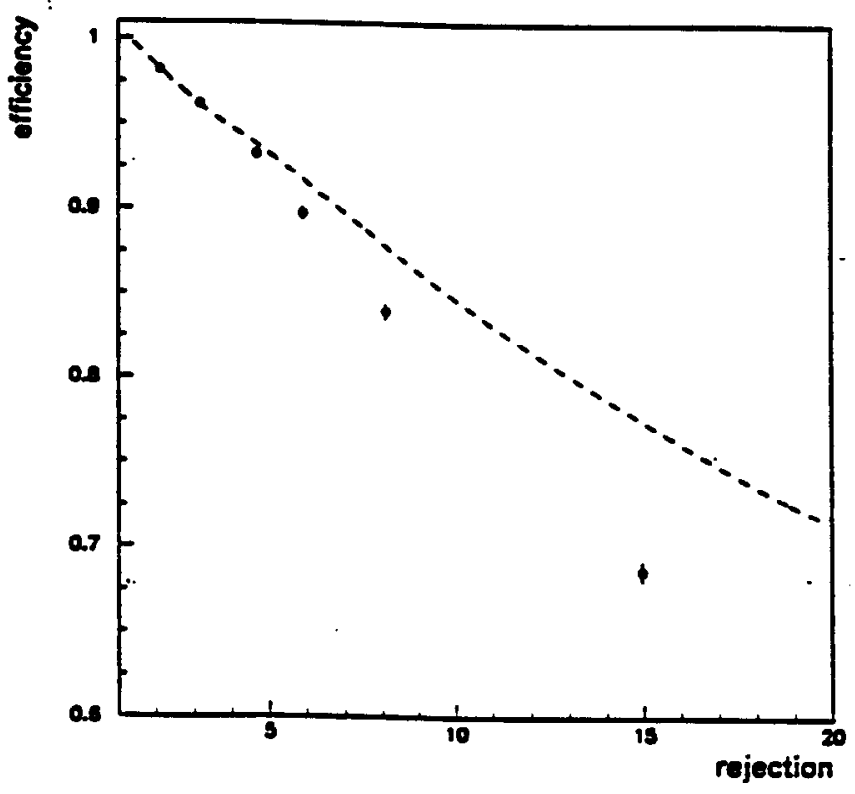

Fig.9 The electron detection efficiency versus rejection power of the minimum bias hadronic background. 


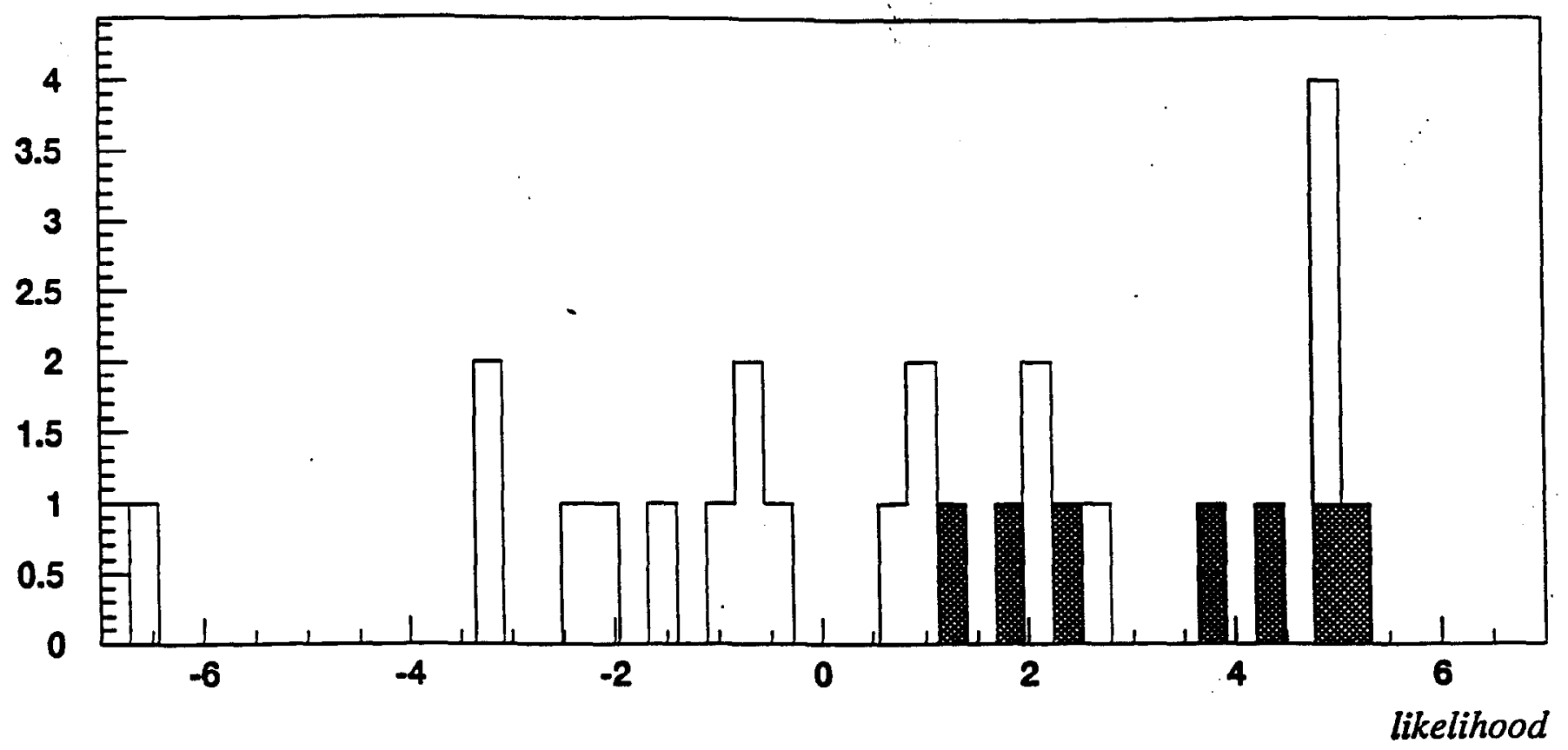

Fig.10 The distribution of the Likelihood function for the electron candidates in the top quark candidate event sample from the D0 colliding beam experiment. The events used in the top quark mass analysis are shown in black. 